\title{
Contribuições de Linha de Produto e Orientação a Serviços no Desenvolvimento de Sistemas de Informação
}

\author{
Roberto dos Santos Rocha, Marcelo Fantinato, Vitor Almeida Barros \\ Escola de Artes, Ciências e Humanidades - Universidade de São Paulo \\ São Paulo - SP, Brasil \\ \{rsrocha, m.fantinato, vitor.almeida.barros\} @usp.br
}

\begin{abstract}
Researchers have sought to identify similarities and differences between Software Product Line and Service Oriented Architecture, both widely used in information systems engineering. This paper aims at presenting the results of an investigation into strategies that join both approaches, using a systematic mapping. We hope it will contribute to understand the needs of information systems engineering in this specific field, making possible to identify aspects that still need further investigation. One of the results being presented is that there already is a large number of papers published in this recent area, though only a few of them are published in journals.
\end{abstract}

Resumo. Pesquisadores têm procurado identificar similaridades e diferenças entre Linha de Produto de Software e Arquitetura Orientada a Serviços, ambas amplamente usadas na engenharia de sistemas de informação. Este artigo busca apresentar os resultados de um mapeamento sistemático realizado sobre estratégias que unam as duas abordagens. Espera-se com este trabalho contribuir ao entendimento das necessidades da área de engenharia de sistemas de informação nesse domínio específico, possibilitando identificar aspectos que ainda necessitam de investigação adicional. Um dos resultados apresentados é que, embora recente, essa área já possui um número bastante grande de artigos publicados, porém ainda bem pouco em periódicos.

\section{Introdução}

A atual complexidade do mundo corporativo tem exigido dinamismo da infraestrutura de Tecnologia da Informação (TI) para oferecer soluções técnicas à condução de negócios. Assim, os Sistemas de Informação (SI) tornaram-se essenciais para ajudar as organizações a atuarem nesse ambiente dinâmico.

Disponibilizar um sistema flexível ao mercado tão cedo quanto possível tornouse um fator crítico de sucesso para a maioria das organizações, tendo em vista que os SI estão relacionados aos processos de negócio e, por sua vez, esses processos estão em constante transformação no ambiente organizacional. A aplicação de técnicas de reúso durante o desenvolvimento de SI pode tornar mais rápida a produção do sistema [Sommerville 2007]. Nesse contexto, Linha de Produto (LP) e Arquitetura Orientada a Serviços (SOA - Service-oriented Architecture) podem fornecer apoio para melhorar a competitividade das organizações, principalmente, se usadas em conjunto.

LP é um conjunto de sistemas que usam software intensivamente, compartilhando um conjunto de características comuns e gerenciadas, que satisfazem as necessidades de um segmento particular de mercado ou missão, e que são desenvolvidos a partir de um conjunto comum de ativos principais e de uma forma 
preestabelecida [Clements and Northrop 2001]. O processo sistemático para desenvolvimento de uma LP possui dois ciclos de vida - Engenharia de Domínio e Engenharia de Aplicação - que usam como artefato base a arquitetura da LP. Em ambos, uma técnica de captura e gerenciamento dos pontos comuns e dos pontos variáveis das propriedades existentes na família de produtos deve ser usada [Gimenes and Travassos 2002]. Uma das técnicas mais usadas ao gerenciamento das variabilidades em uma LP são os modelos de características [Kang and Baik 2010].

SOA é um paradigma arquitetural que trata da integração entre diferentes sistemas, via fornecimento e consumo de serviços eletrônicos, principalmente em um escopo interorganizacional [Alonso et al. 2004]. Os serviços são definidos, invocados e compostos, considerando interfaces bem definidas, podendo ser usado para isso uma terceira parte de apoio - os diretórios de serviço. A principal forma atualmente existente para se implementar o paradigma SOA é a tecnologia de serviços web [Baldam et al. 2007]: componentes de software fracamente acoplados que usam XML para troca de informações com outros aplicativos por meio de padrões bem definidos da internet.

LP e SOA satisfazem uma meta comum de engenharia de SI - reúso - aplicando uma mesma parte de software em diferentes contextos. Princípios de LP ajudam no desenvolvimento de arquiteturas que podem ser reusadas em várias instâncias representantes da família de produtos [Galster 2010]. Por outro lado, com princípios de SOA, é possível desenvolver arquiteturas genéricas para sistemas adaptativos em ambiente heterogêneos que permitem ajustes em tempo de execução. A união das duas abordagens resulta em Linha de Produto Orientadas a Serviços (chamadas normalmente de LPSOA), que pode ser considerada como um conjunto de sistemas orientados a serviços similares que apóia processos de negócio de um domínio específico e pode ser desenvolvida a partir de um núcleo comum de artefatos reusáveis [Northrop et al. 2009].

Um tipo de LP importante ao contexto de LPSOA são as LP Dinâmicas (LPD). Uma LPD produz sistemas capazes de adaptação a mudanças para atender à necessidade do usuário, levando em conta restrições de recursos [Hallsteinsen et al. 2008]. LPD tem sido identificada como uma estratégia promissora para lidar com mudanças a serem realizadas em tempo de execução em aplicações de novos domínios [Burégio et al. 2010]. LPSOA e LPD se relacionam conceitualmente; para Lee e Kotonya (2010), toda LPSOA é LPD, visto que aplicando princípios de SOA desenvolve-se arquiteturas genéricas para sistemas adaptativos em ambiente heterogêneos que permitem ajustes em tempo de execução de forma que a LP torna-se naturalmente uma LPD. Por outro lado, para um conjunto de sistemas, princípios de LP ajudam no desenvolvimento de arquiteturas SOA a serem reusadas em várias instâncias de produto similares.

Visando contribuir com essa área de pesquisa e desenvolvimento da engenharia de SI, foi realizada uma ampla investigação sistemática sobre os trabalhos existentes que apresentam estratégias de aplicação de LPSOA, sejam de artigos que tratam essa ligação de forma explícita ou de forma implícita. Este artigo tem, portanto, o objetivo principal de apresentar os resultados da investigação realizada buscando estratégias existentes de LPSOA, por meio de um mapeamento sistemático (MS). Não é objetivo deste trabalho apresentar descrição de cada um dos trabalhos encontrados no MS.

O restante do artigo compreende: a Seção 2 apresenta trabalhos similares a este; a Seção 3 apresenta a metodologia aplicada; as seções seguintes, da Seção 4 à Seção 7, apresentam os resultados deste MS; e, finalmente, a Seção 8 apresenta as conclusões. 


\section{Trabalhos Relacionados}

No escopo específico de LP, existem já algumas investigações sistemáticas que analisam aspectos particulares de LP, tais como: engenharia de requisitos para LP [Alves, V. et al. 2010]; análise de domínio de LP [Khurum and Gorschek 2009]; gerenciamento de variabilidade de LP [Chen et al. 2009]; e, LPD [Burégio et al. 2010]. O mesmo ocorre ao contexto específico de SOA, como os trabalhos de revisão de Papazoglou et al. (2008) e a RS de Gu e Lago (2009).

Já em relação a união de LP e SOA, por se tratar de um aspecto novo em termos de pesquisa e de aplicação na área de engenharia de SI, as abordagens de LPSOA ainda não tem sido alvo de constantes estudos sistemáticos. Bem recentemente, um estudo preliminar que trata desse aspecto foi publicado em um workshop na área de LP [Murugesupillai et al. 2011]. O estudo realizado pelos autores não segue critérios tão sistemáticos e abrangentes de busca de trabalhos, tais como: apenas foram selecionados trabalhos publicados no período de 2002 a 2010; a string de busca apresentada é restrita; e não utilizam bases de dados de grande abrangência como, por exemplo, o Scopus. Assim, os resultados do MS apresentados aqui são mais amplos e confiáveis para serem usados como referência à comunidade interessada.

\section{Metodologia}

Revisão Sistemática (RS), um dos tipos mais conhecidos de revisões bibliográficas, busca identificar, avaliar e interpretar pesquisas disponíveis relevantes para uma particular área ou questão de pesquisa [Kitchenham 2007], usando uma abordagem replicável, científica e transparente para evitar tendências [Biolchini et al. 2005]. Neste trabalho, foi realizado um Mapeamento Sistemático (MS), usado como uma pré-RS quando ao domínio alvo percebe-se que existem poucas evidências empíricas ou que o tópico é amplo demais [Kitchenham 2007], ambos válidos atualmente para LPSOA.

As etapas iniciais do MS é muito similar a um RS, com a diferença de que não se objetiva como resultado final a interpretação profunda dos trabalhos selecionados mas uma categorização clara da área. O processo de MS adotado consiste dos seguintes passos: (1) definir o escopo do mapeamento, por meio de questões de pesquisa; (2) buscar em bases de dados, por meio de palavras-chaves, a lista de artigos candidatos a serem usados para responder as questões de pesquisa; (3) produzir a lista de artigos relevantes aplicando um filtro com critérios de inclusão/exclusão na lista inicial de artigos candidatos; (4) definir um mecanismo de classificação para poder agrupar os artigos relevantes com base em palavras-chaves recorrentes neles; (5) avaliar o conteúdo dos artigos a ponto de finalmente classificá-los e produzir o MS resultante.

\section{Escopo da Revisão e Artigos Selecionados}

Esta seção apresenta resultados dos três primeiros passos do processo de MS.

\subsection{Questões de Pesquisa}

Como resultado do primeiro passo, as seguintes questões de pesquisa foram definidas considerando a aplicação de abordagens de LPSOA no contexto de Engenharia de SI:

Q1. Quais conceitos/itens de LP estão sendo aplicados no contexto de LPSOA?

Q2. Todas as LPSOA podem ser consideradas dinâmicas? 
Q3. Quais tipos de contribuição estão sendo apresentados pelos trabalhos identificados no contexto de LPSOA?

Q4. Quais métodos de pesquisa estão sendo usados para validar as propostas das abordagens LPSOA identificadas?

\subsection{Seleção de Lista Completa de Artigos}

Para a busca de artigos, a seguinte string de busca foi usada, baseada em palavraschaves derivadas das questões de pesquisa apresentadas na seção anterior:

("product line" OR "product family" OR "product families" OR "spl" OR "domain engineering" OR "domain analysis" OR "feature model" OR "variability" OR "variabilities") AND

("soc" OR "soa" OR "service-oriented" OR "service-orientation" OR "service-based" OR "web service" OR "electronic service" OR "e-service")

Essa busca teve o objetivo de encontrar trabalhos que estivessem relacionados ao escopo de estratégias LPSOA não apenas de forma explícita mas também de forma implícita. Por isso palavras-chaves como "domain engineering" e "variability" foram usadas, além da esperada "product line", visto que as duas primeiras também fazem parte do escopo de LP.

Essa string de busca foi submetida ao mecanismo de busca do Scopus (http://www.scopus.com) e do DBLP (The DBLP Computer Science Bibliography) (http://www.dblp.org/db/), que incluem registros das principais conferências e dos principais periódicos publicados em bibliotecas digitais tais como Springer, ACM e IEEE e para outros importantes veículos indexados. Uma análise demonstrou que o uso da base WebOfScience (http://apps.isiknowledge.com/) seria redundante. Com o resultado, foram realizadas leitura dos títulos e das palavras-chave, e os trabalhos resultantes claramente irrelevantes às questões investigadas foram excluídos.

Foram considerados também todos os artigos publicados no período de 20072009 do workshop SOAPL (Workshop on Service-Oriented Architectures and Software Product Lines), por ser o único fórum dedicado a essa área de pesquisa.

Ao todo, identificou-se 998 artigos como a lista inicial de "todos os artigos".

\subsection{Seleção de Artigos Relevantes}

Para filtrar a lista e selecionado apenas a lista de "artigos relevantes", os seguintes Critérios de Inclusão (CI) e Critérios de Exclusão (CE) foram aplicados:

- CI 1: artigo publicado entre 2001 e 2011, visto que não foram identificados trabalhos nesta área em uma busca prévia a este MS para antes de 2001;

- CI 2: artigo está disponível na web ou é enviado pelos autores por e-mail;

- CI 3: artigo é apresentado integralmente na língua inglesa, e não apenas resumo;

- $\boldsymbol{C E}$ 1: artigo não pertence ao domínio da engenharia de SI;

- $\quad \boldsymbol{C E} 2$ 2: LP ou SOA não faz parte do escopo principal do artigo e os termos são mencionados apenas de forma geral nos resumos ou nas sentenças introdutórias;

- $\boldsymbol{C E}$ 3: artigo apresenta uma abordagem muito específica à uma fase da engenharia de SI, como verificação, validação e testes;

- $\boldsymbol{C E}$ 4: publicações não revisadas por pares (non-peer reviewed), tais como relatórios técnicos, livros e descrições de workshop. 
Os critérios de inclusão e de exclusão foram aplicados com base em uma leitura inicial dos artigos, incluindo mais do que o resumo e a conclusão como sugerido pela metodologia apresentada na Seção 3, para conferir maior confiabilidade ao resultado. Esse processo foi documento, com o auxílio de uma planilha eletrônica. As possíveis dúvidas sobre a inclusão/exclusão de um artigo foram resolvidas pelo segundo pesquisador, por ser o mais experiente do grupo.

Como resultado final, foram selecionados 98 "trabalhos relevantes", 87 de conferências (Tabela 1) e 11 de periódicos (Tabela 2). Como contribuição adicional deste artigo, a lista de referências desses trabalhos está disponível em um repositório, localizado virtualmente (http://lpsoa.users.sourceforge.net) devido à limitação de espaço.

Tabela 1. Lista final de "trabalhos relevantes" (Conferências)

\begin{tabular}{|c|c|c|c|c|}
\hline Ano & ID & Artigos & ID & Artigos \\
\hline 2003 & $\mathrm{C} 01$ & [Aßmann 2003] & $\mathrm{C} 02$ & [Diaz et al. 2003] \\
\hline 2004 & $\mathrm{C} 03$ & [Topaloglu and Capilla 2004] & $\mathrm{C} 04$ & [Zhao et al. 2004] \\
\hline 2005 & $\begin{array}{l}\mathrm{C} 05 \\
\mathrm{C} 06\end{array}$ & $\begin{array}{l}\text { [Altintas and Cetin 2005] } \\
\text { [Capilla and Topaloglu 2005] }\end{array}$ & $\begin{array}{l}\mathrm{C} 07 \\
\mathrm{C} 08\end{array}$ & $\begin{array}{l}\text { [Gomaa and Saleh 2005] } \\
\text { [Jiang et al. 2005] }\end{array}$ \\
\hline 2006 & $\begin{array}{l}\mathrm{C} 09 \\
\mathrm{C} 10\end{array}$ & $\begin{array}{l}\text { [Gomaa and Saleh 2006] } \\
\text { [Schulz-Hofen and Golega 2006] }\end{array}$ & $\mathrm{C} 11$ & [Zhang and Kunz 2006] \\
\hline 2007 & $\begin{array}{l}\text { C12 } \\
\text { C13 } \\
\text { C14 } \\
\text { C15 } \\
\text { C16 } \\
\text { C17 }\end{array}$ & $\begin{array}{l}\text { [Chang 2007] } \\
\text { [Chang and Kim 2007] } \\
\text { [Fantinato et al. 2007] } \\
\text { [Gruler et al. 2007] } \\
\text { [Helferich et al. 2007a] } \\
\text { [Helferich et al. 2007b] } \\
\end{array}$ & $\begin{array}{l}\text { C18 } \\
\text { C19 } \\
\text { C20 } \\
\text { C21 } \\
\text { C22 } \\
\text { C23 }\end{array}$ & $\begin{array}{l}\text { [Lee et al. 2007] } \\
\text { [Raatikainen et al. 2007] } \\
\text { [Segura et al. 2007] } \\
\text { [Trujillo et al. 2007] } \\
\text { [Wada et al. 2007] } \\
\text { [Ye et al. 2007] }\end{array}$ \\
\hline 2008 & $\begin{array}{l}\mathrm{C} 24 \\
\mathrm{C} 25 \\
\mathrm{C} 26 \\
\mathrm{C} 27 \\
\mathrm{C} 28 \\
\mathrm{C} 29 \\
\mathrm{C} 30 \\
\mathrm{C} 31 \\
\mathrm{C} 32 \\
\end{array}$ & $\begin{array}{l}\text { [Acher et al. 2008] } \\
\text { [Adam and Doerr 2008] } \\
\text { [Apel et al. 2008] } \\
\text { [Bartholdt et al. 2008] } \\
\text { [Boffoli et al. 2008] } \\
\text { [Budny et al. 2008] } \\
\text { [Clotet et al. 2008] } \\
\text { [Gimenes et al. 2008] } \\
\text { [Günther and Berger 2008] }\end{array}$ & $\begin{array}{l}\text { C33 } \\
\text { C34 } \\
\text { C35 } \\
\text { C36 } \\
\text { C37 } \\
\text { C38 } \\
\text { C39 } \\
\text { C40 }\end{array}$ & $\begin{array}{l}\text { [Harhurin and Hartmann 2008] } \\
\text { [Kang et al. 2008] } \\
\text { [Kim and Doh 2008] } \\
\text { [Lee et al. 2008] } \\
\text { [Li et al. 2008] } \\
\text { [Mietzner and Leymann 2008] } \\
\text { [Rusk and Gasevic 2008] } \\
\text { [Shokry and Babar 2008] }\end{array}$ \\
\hline 2009 & $\begin{array}{l}\text { C41 } \\
\text { C42 } \\
\text { C43 } \\
\text { C44 } \\
\text { C45 } \\
\text { C46 } \\
\text { C47 }\end{array}$ & $\begin{array}{l}\text { [Asadi et al. 2009] } \\
\text { [Boffoli et al. 2009] } \\
\text { [Istoan et al. 2009] } \\
\text { [Kotonya et al. 2009] } \\
\text { [Lee et al. 2009] } \\
\text { [Medeiros et al. 2009] } \\
\text { [Mohabbati et al. 2009] }\end{array}$ & $\begin{array}{l}\text { C48 } \\
\text { C49 } \\
\text { C50 } \\
\text { C51 } \\
\text { C52 } \\
\text { C53 }\end{array}$ & $\begin{array}{l}\text { [Nunes et al. 2009] } \\
\text { [Parra et al. 2009] } \\
\text { [Schäfer and Dolog 2009] } \\
\text { [Smith and Lewis 2009] } \\
\text { [Sun, H. et al. 2009] } \\
\text { [Xiao et al. 2009] }\end{array}$ \\
\hline 2010 & $\begin{array}{l}\text { C54 } \\
\text { C55 } \\
\text { C56 } \\
\text { C57 } \\
\text { C58 } \\
\text { C59 } \\
\text { C60 }\end{array}$ & $\begin{array}{l}\text { [Aiello et al. 2010] } \\
\text { [Galster 2010] } \\
\text { [Kang and Baik 2010] } \\
\text { [Kattepur et al. 2010] } \\
\text { [Medeiros et al. 2010a] } \\
\text { [Medeiros et al. 2010b] } \\
\text { [Nguyen and Colman 2010] }\end{array}$ & $\begin{array}{l}\text { C61 } \\
\text { C62 } \\
\text { C63 } \\
\text { C64 } \\
\text { C65 } \\
\text { C66 }\end{array}$ & $\begin{array}{l}\text { [Park et al. 2010] } \\
\text { [Ribeiro et al. 2010] } \\
\text { [Souer and Joor 2010] } \\
\text { [Stollberg and Muth 2010] } \\
\text { [Sun, Z. et al. 2010] } \\
\text { [Yu et al. 2010] }\end{array}$ \\
\hline 2011 & $\begin{array}{l}\text { C67 } \\
\text { C68 } \\
\text { C69 } \\
\text { C70 } \\
\text { C71 } \\
\text { C72 } \\
\text { C73 } \\
\text { C74 } \\
\text { C75 } \\
\text { C76 } \\
\text { C77 }\end{array}$ & $\begin{array}{l}\text { [Abotsi et al. 2011] } \\
\text { [Abu-Matar and Gomaa 2011a] } \\
\text { [Abu-Matar and Gomaa 2011b] } \\
\text { [Alférez and Pelechano 2011a] } \\
\text { [Alférez and Pelechano 2011b] } \\
\text { [Dhungana et al. 2011a] } \\
\text { [Dhungana et al. 2011b] } \\
\text { [Dobrica and Ovaska 2011] } \\
\text { [Galster and Eberlein 2011] } \\
\text { [Gomaa and Hashimoto 2011] } \\
\text { [Khan et al. 2011] }\end{array}$ & $\begin{array}{l}\text { C78 } \\
\text { C79 } \\
\text { C80 } \\
\text { C81 } \\
\text { C82 } \\
\text { C83 } \\
\text { C84 } \\
\text { C85 } \\
\text { C86 } \\
\text { C87 }\end{array}$ & $\begin{array}{l}\text { [Kulkarni and Barat 2011b] } \\
\text { [Mohabbati et al. 2011a] } \\
\text { [Mohabbati et al. 2011b] } \\
\text { [Nascimento et al. 2011] } \\
\text { [Nguyen et al. 2011] } \\
\text { [Park and Yeom 2011] } \\
\text { [Ribeiro et al. 2011] } \\
\text { [Serajzadeh and Shams 2011] } \\
\text { [Yeom et al. 2011] } \\
\text { [Ying et al. 2011] }\end{array}$ \\
\hline
\end{tabular}


Tabela 2. Lista final de "trabalhos relevantes" (Periódicos)

\begin{tabular}{|c|l|l|c|l|}
\hline Ano & \multicolumn{1}{|c|}{ ID } & \multicolumn{1}{|c|}{ Artigos } & ID & \multicolumn{1}{c|}{ Artigos } \\
\hline 2008 & P01 & [Fábio et al. 2008] & P02 & [Karam et al. 2008] \\
\hline 2009 & P03 & [Koning et al. 2009] & P04 & [White et al. 2009] \\
\hline 2010 & $\begin{array}{l}\text { P05 } \\
\text { P06 }\end{array}$ & $\begin{array}{l}\text { [Lee and Kotonya 2010] } \\
\text { [Lee et al. 2010] }\end{array}$ & P07 & [Sun, C. et al. 2010] \\
\hline 2011 & $\begin{array}{l}\text { P08 } \\
\text { P09 }\end{array}$ & $\begin{array}{l}\text { P10 } 10 \\
\text { [Kulkalves et al. 2011] }\end{array}$ & $\begin{array}{l}\text { [Park et al. 2011] } \\
\text { [Wu and Wei 2011] }\end{array}$ \\
\hline
\end{tabular}

Todos esses trabalhos passaram por uma leitura mais aprofundada, buscando principalmente responder as questões de pesquisa. Por limitação de espaço, não são apresentados resumos desses trabalhos, nem mesmo daqueles considerados mais representativos. Tal descrição seria considerada um objetivo secundário deste artigo, cujo principal objetivo é oferecer uma visão consolidada para que a comunidade interessada busque os trabalhos que possam mais lhe interessar.

\section{Classificação de Trabalhos Relevantes}

Como esquema de classificação dos trabalhos deste MS, adotou-se o mecanismo de facetas proposto por Petersen et al. (2008) e Pietro-Diaz e Freeman (1987), em função de sua flexibilidade. Como ponto de partida, as duas primeiras facetas foram criadas com base nos exemplos de Petersen et al. (2008), adaptando-as quando necessário. As classificações foram conduzidas pelo primeiro e terceiro autores e validadas pelo segundo, por ser o mais experiente.

A Faceta 1, chamada "Foco de Pesquisa" (descrita na Tabela 3), categoriza os trabalhos em função de conceitos (itens) inerentes a LP, identificando quais deles são aplicados no contexto de LPSOA, em cada trabalho selecionado. Especificamente, essa faceta ajuda responder as questões de pesquisa Q1 e Q2 apresentadas na Seção 4.1.

\section{Tabela 3. Foco de Pesquisa (Faceta 1)}

\begin{tabular}{|l|l|}
\hline \multicolumn{1}{|c|}{ Categoria } & \multicolumn{1}{c|}{ Descrição } \\
\hline Engenharia de Domínio & Engenharia de Domínio é enfocada explicitamente no contexto de LPSOA \\
\hline Engenharia de Aplicação & Engenharia de Aplicação é enfocada explicitamente no contexto de LPSOA. \\
\hline Proposta de Arquitetura & Uma arquitetura é proposta explicitamente no contexto de LPSOA. \\
\hline Variabilidade & Variabilidades são tratadas explicitamente no contexto de LPSOA. \\
\hline Modelo de Característica & $\begin{array}{l}\text { Modelos de características são usadas explicitamente no tratamento de variabilidades no } \\
\text { contexto de LPSOA. }\end{array}$ \\
\hline Domínio Específico & Um domínio de aplicação específico é abordado explicitamente no contexto de LPSOA. \\
\hline LPD & O domínio de LPD é abordado explicitamente no contexto de LPSOA. \\
\hline
\end{tabular}

A Faceta 2, chamada "Tipo de Contribuição" (descrita na Tabela 4), categoriza os trabalhos em função das contribuições propostas. Essa faceta ajuda responder Q3.

Tabela 4. Tipo de Contribuição (Faceta 2)

\begin{tabular}{|l|l|}
\hline \multicolumn{1}{|c|}{ Categoria } & \multicolumn{1}{c|}{ Descrição } \\
\hline $\begin{array}{l}\text { Metodologia de } \\
\text { Desenvolvimento }\end{array}$ & $\begin{array}{l}\text { Trabalho que apresenta uma metodologia de desenvolvimento ao contexto de LPSOA, } \\
\text { descrevendo atividades ou ações e seus fluxos de trabalho. Incluem métodos, regras ou diretrizes } \\
\text { de como as atividades devem ser realizadas. }\end{array}$ \\
\hline $\begin{array}{l}\text { Discussão } \\
\text { Conceitual }\end{array}$ & $\begin{array}{l}\text { Trabalho que apresenta taxonomias, questões de identificação, ou relatos de experiência com } \\
\text { caracterização de desafios ou problemas, no contexto de LPSOA. }\end{array}$ \\
\hline $\begin{array}{l}\text { Modelo } \\
\text { Conceitual }\end{array}$ & $\begin{array}{l}\text { Trabalho que apresenta um modelo conceitual ao contexto de LPSOA, original ou extensão de } \\
\text { um existente, e apresentado na forma de uma representação do mundo real em um nível de } \\
\text { abstração diferente no qual detalhes são omitidos . }\end{array}$ \\
\hline Ferramenta & Trabalho que apresenta uma ferramenta computacional para abordagens de LPSOA. \\
\hline Algoritmo & Trabalho que apresenta um ou mais algoritmos para lidar com aspectos de LPSOA. \\
\hline
\end{tabular}


A Faceta 3, chamada "Tipo de Pesquisa" (descrita na Tabela 5), categoriza os trabalhos em função do procedimento metodológico usado, partindo do proposto por Wieringa et al. (2005). Essa faceta ajuda responder Q4.

Tabela 5. Tipo de Pesquisa (Faceta 3)

\begin{tabular}{|l|l|}
\hline Categoria & \multicolumn{1}{c|}{ Descrição } \\
\hline $\begin{array}{l}\text { Proposta de } \\
\text { Solução }\end{array}$ & $\begin{array}{l}\text { Trabalho que propõe uma solução para um problema relacionado à LPSOA, que pode ser tanto } \\
\text { nova ou uma extensão significativa de uma abordagem existente. Nenhuma validação formal é } \\
\text { realizada. Os benefícios em potencial e a aplicabilidade da solução são indicados por um } \\
\text { pequeno exemplo ou uma boa argumentação. }\end{array}$ \\
\hline $\begin{array}{l}\text { Validação da } \\
\text { Pesquisa }\end{array}$ & $\begin{array}{l}\text { Trabalho que investiga uma proposta de solução para LPSOA que ainda não foi implementada } \\
\text { na prática industrial. Geralmente, as abordagens são avaliadas por meio de: experimentos, } \\
\text { simulação, prototipagem, e prova matemática de propriedades, entre outros. }\end{array}$ \\
\hline $\begin{array}{l}\text { Avaliação da } \\
\text { Pesquisa }\end{array}$ & $\begin{array}{l}\text { Trabalho que apresenta resultados da avaliação conduzida sobre uma abordagem para LPSOA já } \\
\text { implementada na prática industrial. A avaliação apresenta como a abordagem é aplicada na } \\
\text { prática (a implementação da solução) e quais são as consequências da implementação em termos } \\
\text { de vantagens e desvantagens (avaliação da execução). }\end{array}$ \\
\hline Artigo Conceitual & Trabalho que apresenta uma taxonomia para LPSOA ou um framework conceitual para LPSOA. \\
\hline $\begin{array}{l}\text { Relato de } \\
\text { Experiência }\end{array}$ & $\begin{array}{l}\text { Trabalho que apresenta uma descrição de alguma experiência prática, normalmente na indústria, } \\
\text { relacionada com o uso de uma abordagem de LPSOA. Normalmente oferece como principal } \\
\text { resultado um conjunto de lições aprendidas. }\end{array}$ \\
\hline
\end{tabular}

O esquema de classificação definido permite que um mesmo trabalho seja classificado, para uma mesma faceta, em mais de uma categoria. Por exemplo, à Faceta 1, um único trabalho pode apresentar detalhes de LPSOA relacionados a gerenciamento da variabilidade, engenharia de domínio e engenharia de aplicação; sendo, portanto, classificados em três categorias.

\section{Mapa Sistemático}

Esta seção apresenta os resultados finais do MS. Primeiramente, a distribuição temporal dos trabalhos relevantes, separados por tipo de veículo, é apresentada na Figura 1. Destaca-se que apenas $11 \%$ são publicados em periódicos e que, no total, $77 \%$ foram publicados nos últimos quatro anos, sendo $25 \%$ no último ano. Esses são indicativos de que a área de LPSOA encontra-se em estágio inicial e ainda em ganho de maturidade.

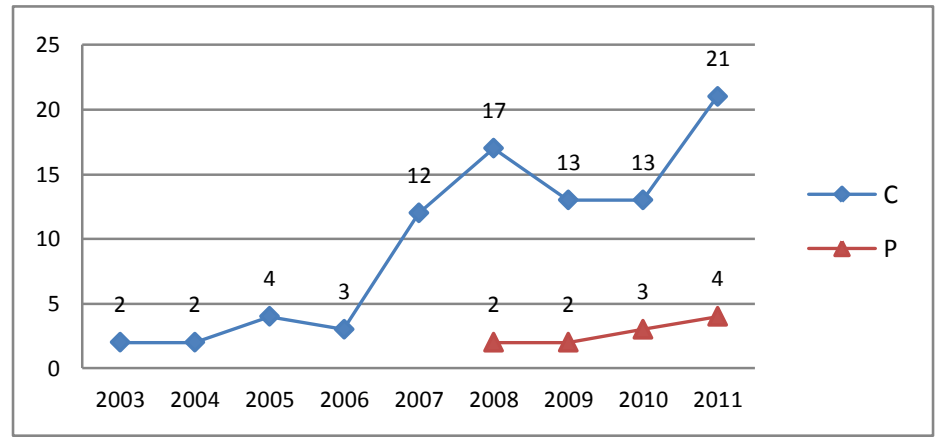

Figura 1. Distribuição temporal dos estudos (C: Conferência / P: Periódico)

A Figura 2 apresenta a distribuição dos artigos pelos veículos de publicação. Para conferências, há grande concentração na SPLC (Software Product Line Conference) (20\%) e em um de seus workshops, o SOAPL (16\%), enquanto o restante está pulverizado por várias outras. Já para os periódicos, considerando o pequeno número de publicações (apenas 11\% do total), não há nenhum padrão identificado. 


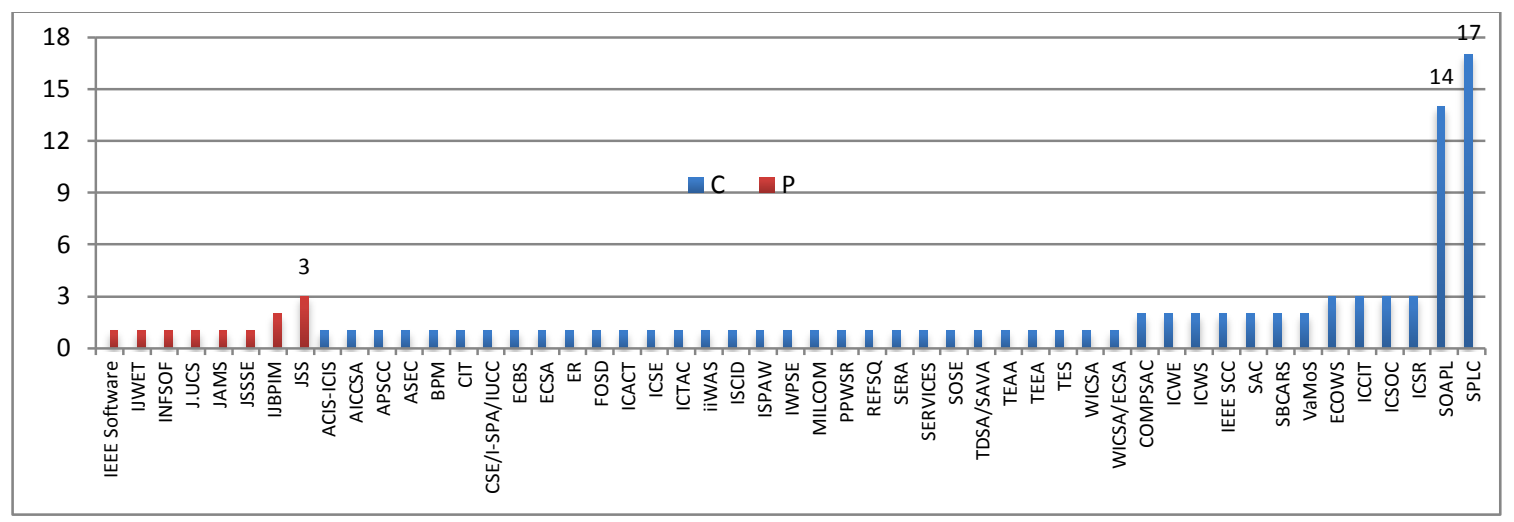

Figura 2. Distribuição dos estudos por veículos (C: Conferência / P: Periódico)

As tabelas a seguir (Tabelas 6, 7 e 8) apresentam a classificação geral respectivamente em cada uma das três facetas (Faceta 1, 2 e 3).

Tabela 6. Trabalhos por Foco de Pesquisa (Faceta 1)

\begin{tabular}{|c|c|}
\hline Categoria & Trabalhos \\
\hline $\begin{array}{l}\text { Engenharia de } \\
\text { Domínio }\end{array}$ & $\begin{array}{l}\text { C04,C06,C09,C10,C15,C17,C23,C25,C39,C40,C41,C43,C44,C45,C48,,C50,C52,C55,C59,C60, } \\
\text { C63,C66,C67,C70,C71,C79,C80,C84,C85,P02, P05,P06,P08 }\end{array}$ \\
\hline $\begin{array}{l}\text { Engenharia de } \\
\text { Aplicação }\end{array}$ & $\begin{array}{l}\text { C06,C09,C10,C15,C17,C23,C25,C41,C50,C52,C55,C59,C60,C63,C66,C67,C70,C71,C79,C80, } \\
\text { C85,P05,P06,P08 }\end{array}$ \\
\hline $\begin{array}{l}\text { Proposta de } \\
\text { Arquitetura }\end{array}$ & $\mathrm{C} 05, \mathrm{C} 11, \mathrm{C} 17, \mathrm{C} 23, \mathrm{C} 29, \mathrm{C} 36, \mathrm{C} 40, \mathrm{C} 56, \mathrm{C} 58, \mathrm{C} 59, \mathrm{C} 66, \mathrm{C} 72, \mathrm{C} 74, \mathrm{P} 05, \mathrm{P} 06$ \\
\hline Variabilidade & $\begin{array}{l}\text { C02,C03,C06,C07,C08,C10,C12,C13,C14,C15,C23,C25,C28,C30,C33,C35,C36,C37,C38,C40, } \\
\text { C41,C43,C48,C50,C52,C54,C55,C57,C58,C59,C60,C61,C62,C63,C64,C66,C67,C68,C69,C70, } \\
\text { C71,C72,C73,C74,C75,C76,C77,C78,C79,C80,C81,C82,C83,C84,C86,C87,P03,P04,P05,P06, } \\
\text { P07,P08,P09,P10,P11 }\end{array}$ \\
\hline $\begin{array}{l}\text { Modelo de } \\
\text { Característica }\end{array}$ & $\begin{array}{l}\text { C02,C07,C09,C14,C15,C22,C26,C27,C31,C34,C35,C36,C43,C44,C45,C50,C55,C56,C57,C59, } \\
\text { C60,C61,C63,C68,C69,C70,C71,C72,C74,C76,C79,C80,C81,C83,C87,P01,P04,P05,P06,P08,P11 }\end{array}$ \\
\hline Domínio Específico & $\mathrm{C} 01, \mathrm{C} 04, \mathrm{C} 28, \mathrm{C} 29, \mathrm{C} 30, \mathrm{C} 31, \mathrm{C} 39, \mathrm{C} 40, \mathrm{C} 41, \mathrm{C} 43, \mathrm{C} 48, \mathrm{C} 49, \mathrm{C} 53, \mathrm{C} 66$ \\
\hline LPD & $\mathrm{C} 05, \mathrm{C} 09, \mathrm{C} 10, \mathrm{C} 12, \mathrm{C} 40, \mathrm{C} 43, \mathrm{C} 49, \mathrm{C} 50, \mathrm{C} 54, \mathrm{C} 55, \mathrm{C} 58, \mathrm{C} 60, \mathrm{C} 66, \mathrm{C} 67, \mathrm{C} 71, \mathrm{C} 76, \mathrm{C} 81, \mathrm{C} 82, \mathrm{P} 04, \mathrm{P} 05$ \\
\hline
\end{tabular}

Tabela 7. Trabalhos por Tipo de Contribuição (Faceta 2)

\begin{tabular}{|c|c|}
\hline Categoria & Trabalhos \\
\hline $\begin{array}{l}\text { Metodologia de } \\
\text { Desenvolvimento }\end{array}$ & $\begin{array}{l}\text { C02,C04,C06,C07,C08,C09,C10,C11,C12,C13,C14,C15,C22,C23,C25,C28,C30,C31,C33,C34, } \\
\text { C35,C36,C38,C40,C41,C44,C45,C50,C52,C56,C57,C58,C59,C60,C61,C63,C64,C65,C66,C67, } \\
\text { C68,C69,C70,C71,C72,C74,C76,C81,C83,C84,C86,C87,P01,P03,P04,P06,P07,P08 }\end{array}$ \\
\hline Discussão Conceitual & $\mathrm{C} 01, \mathrm{C} 02, \mathrm{C} 03, \mathrm{C} 07, \mathrm{C} 08, \mathrm{C} 13, \mathrm{C} 17, \mathrm{C} 25, \mathrm{C} 26, \mathrm{C} 27, \mathrm{C} 33, \mathrm{C} 37, \mathrm{C} 54, \mathrm{C} 62, \mathrm{C} 75, \mathrm{C} 77, \mathrm{C} 78, \mathrm{C} 82, \mathrm{P} 05, \mathrm{P} 09$ \\
\hline Modelo Conceitual & $\mathrm{C} 05, \mathrm{C} 36, \mathrm{C} 39, \mathrm{C} 49, \mathrm{C} 53, \mathrm{C} 55, \mathrm{C} 65, \mathrm{P} 02$ \\
\hline Ferramenta & $\begin{array}{l}\text { C10,C23,C27,C28,C29,C30,C38,C39,C43,C44,C45,C49,C53,C54,C64,C66,C71,C72,C73,C80, } \\
\text { C83,C85,P03,P04,P07,P10 }\end{array}$ \\
\hline Algoritmo & C50,C52,C53,C79,C85,P11 \\
\hline
\end{tabular}

Tabela 8. Trabalhos por Tipo de Pesquisa (Faceta 3)

\begin{tabular}{|l|l|}
\hline \multicolumn{1}{|c|}{ Categoria } & \multicolumn{1}{c|}{ Trabalhos } \\
\hline \multirow{2}{*}{ Proposta de Solução } & $\begin{array}{l}\text { C01,C02,C03,C05,C06,C07,C08,C09,C10,C13,C15,C23,C25,C26,C28,C30,C31,C33,C34, } \\
\text { C36,C38,C39,C40,C41,C43,C44,C45,C48,C50,C52,C53,C54,C55,C56,C58,C61,C64,C65, } \\
\text { C66,C68,C69,C72,C73,C76,C78,C81,C82,C83,C85,C86,C87,P01,P05,P06,P07,P09 }\end{array}$ \\
\hline Validação da Pesquisa & $\begin{array}{l}\text { C04,C08,C11,C12,C15,C28,C36,C37,C38,C49,C52,C59,C60,C62,C63,C67,C70,C71,C74, } \\
\text { C75,C79,C80,P03,P04,P08,P10,P11 }\end{array}$ \\
\hline Avaliação da Pesquisa & $\mathrm{C} 02, \mathrm{C} 57, \mathrm{C} 58, C 84, P 02$ \\
\hline Artigo Conceitual & $\mathrm{C} 17$ \\
\hline Relato de Experiência & $\mathrm{C} 14, \mathrm{C} 27, \mathrm{C} 29$ \\
\hline
\end{tabular}


Nas subseções a seguir são apresentados detalhamentos dos resultados das classificações das três facetas.

\subsection{Foco de Pesquisa}

A Figura 3 apresenta a distribuição de trabalhos por foco de pesquisa (Faceta 1), enfatizando a ordem de ocorrência dos conceitos/itens de LP. Tratamento de variabilidade é o item mais presente nos trabalhos avaliados (em 66\% dos trabalhos); sendo que modelos de características aparecem em $42 \%$ do total de trabalhos, representando $32 \%$ dos trabalhos que apresentam tratamento de variabilidade. Em relação às fases do ciclo de vida de LP, Engenharia de Domínio está mais presente nos trabalhos avaliados (em 34\%) do que Engenharia de Aplicação (em 24\%); e por uma dependência natural, todos os trabalhos que envolvem Engenharia de Aplicação também envolvem Engenharia de Domínio. Apenas 20\% dos trabalhos tratam especificamente de conceitos dinâmicos no contexto de LPSOA. Apesar da importância da arquitetura como um item em LP, apenas $15 \%$ dos trabalhos avaliados nesta apresentam uma proposta de arquitetura para LPSOA. Por fim, apenas 14\%, apresentam propostas no contexto de LPSOA para domínios específicos.

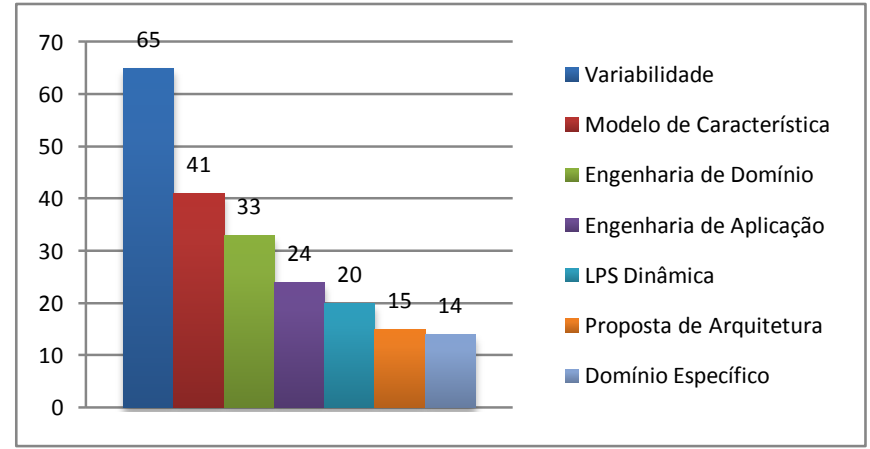

Figura 3. Distribuição dos trabalhos por foco de pesquisa (Faceta 1)

\subsection{Tipo de Contribuição e Tipo de Pesquisa}

A Figura 4 apresenta a distribuição cruzada dos trabalhos por "Tipo de Contribuição" (Faceta 2, no eixo horizontal) e "Tipo de Pesquisa" (Faceta 3, no eixo vertical). O número dentro de cada círculo nas interseções representa o número de trabalhos que se enquadram em cada classificação bidimensional e o tamanho do círculo representa proporcionalmente o número de trabalho em cada interseção.

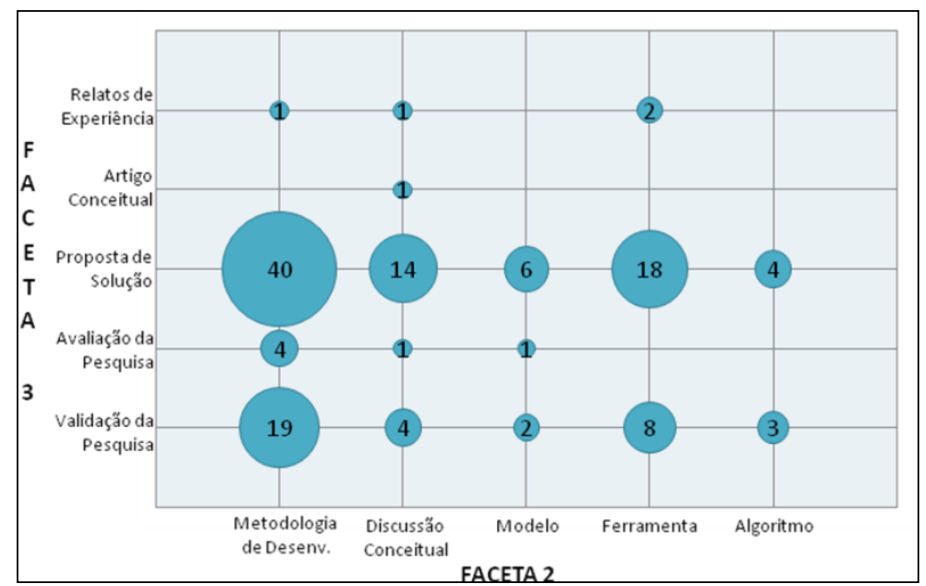

Figura 4. Distribuição de artigos por "Tipo de Contribuição" e "Tipo de Pesquisa" 
Em relação ao "tipo de pesquisa", o maior conjunto de contribuições no contexto de LPSOA refere-se a "Proposta de Solução", totalizando 82 de 129 ocorrências (64\%). Em relação ao "tipo de contribuição", a maior parte das contribuições refere-se a "Metodologia de Desenvolvimento", totalizando 64 de 129 ocorrências (50\%).

Analisando ambas as facetas, a maior intersecção são trabalhos que apresentem uma "Proposta de Solução" relacionadas a uma "Metodologia de Desenvolvimento" (40 trabalhos). Na sequência, as próxima maiores intersecções são trabalhos que apresentam uma "Metodologia de Desenvolvimento" e "Validação da Pesquisa" (19 trabalhos) e "Ferramenta" para apoiar um ou mais aspecto da "Proposta de Solução" (18 trabalhos).

\section{Avaliação Geral}

Os dados apresentados na seção anterior permitem responder, pelo menos parcialmente, as quatro questões de pesquisa apresentadas na Seção 4.1 como norteadoras deste MS.

Em relação à questão Q1 (Quais conceitos de LP estão sendo aplicados no contexto de LPSOA), os sete itens de LP buscados foram encontrados nos trabalhos avaliados, com destaque "variabilidade" e "engenharia de domínio" que são dois dos conceitos mais importantes em LP. A baixa ocorrência do item "arquitetura" chama a atenção visto a sua grande importância tanto em LP quanto em SOA.

Em relação à questão Q2 (Todas as LPSOA podem ser consideradas dinâmicas), apenas em uma pequena parte dos trabalhos avaliados $(20 \%)$ foram encontrados de forma explícita conceitos de LPD, o que não corresponde, pelo menos diretamente, à hipótese de Lee e Kotonya (2010) de que toda LPSOA é uma LPD.

Em relação à questão Q3 (Quais tipos de contribuição estão sendo apresentados pelos trabalhos no contexto de LPSOA identificados), a grande maioria dos trabalhos avaliados tem contribuído com a apresentação de "Metodologia de desenvolvimento", seguida por "Ferramenta" e por "Discussão Conceitual".

Em relação à questão Q4 (Quais métodos de pesquisa estão sendo usados para validar as propostas das abordagens LPSOA identificadas), a grande maioria dos trabalhos avaliados apresenta uma "Proposta de Solução", incluindo normalmente apenas exemplos de aplicação. Poucos trabalhos apresentam uma "Validação da Pesquisa" ou uma "Avaliação da Pesquisa", de forma isolada ou juntamente com a "Proposta de Solução", o que leva a considerar ainda essa uma área de pesquisa recente.

Como um resumo das ameaças à validade deste MS, já considerando validade de conclusão, validade interna, validade de construção e validade externa, têm-se: falta de representação padrão para conteúdo de informação de artigos científicos em engenharia de SI; alguns critérios de inclusão ou de exclusão assim como os esquemas de classificação podem ser considerados subjetivos, dificultando a reprodução do MS por outros pesquisadores, embora buscou-se a minimização do efeito dessa subjetividade pela confirmação dos três autores envolvidos neste MS; e, o grande número de trabalhos retornados para análise possibilita que uma análise final menos confiável seja resultante.

\section{Conclusão}

Este trabalho de investigação apresentou uma caracterização geral, porém importante à área de Engenharia de SI, da pesquisa relacionada a abordagens de LPSOA. Com ela, foi possível reconhecer que já há um grande número de trabalhos de pesquisa sendo 
realizados na área de LP direcionados a SOA, porém ainda sem atingir uma maturidade de trabalhos sólidos, ou seja, trabalhos usados na prática industrial e validados com base em experimentações formais. Grande evidência disso é o alto número de publicações realizadas apenas no último triênio e ainda poucas publicações em periódicos.

A partir deste MS realizado, espera-se continuar o trabalho de análise da área de LPSOA, realizando possivelmente uma RS buscando responder questões de pesquisa mais avançadas. Um exemplo de questão seria validar a hipótese de que realmente toda LPSOA é uma LPD, o que não foi encontrado de forma explícita na grande maioria dos trabalhos avaliados neste trabalho. Outro objetivo para uma RS é a identificação de limitações dos estudos, não realizado neste MS.

\section{Agradecimentos}

À Capes (Coordenação de Aperfeiçoamento de Pessoal de Nível Superior) e à Fapesp (Fundo de Amparo à Pesquisa do Estado de São Paulo) pelo apoio financeiro. À UFBA (Universidade Federal da Bahia) pelo apoio e financiamento a um dos autores. À Prof ${ }^{a}$. Dra. Fátima L. S. Nunes (EACH-USP) pelo auxílio na elaboração deste trabalho.

\section{Referências}

Alonso, G., Casati, F., Kuno, H. and Machiraju, V. (2004). Web Services: Concepts, Architectures and Applications, Springer, $1^{\text {st }}$ edition.

Alves, V., Niu, N., Alves, C. and Valença, G. (2010). Requirements engineering for software product lines: A systematic literature review. Information and Software Technology, v. 52, n. 8, pages 806-820.

Baldam, R. et al. (2007). Gestão de Processos de Negócios: BPM - Business Process Management, Érica, $2^{a}$ edição.

Biolchini, J., Mian, P. G., Natali, A. C. C. and Travassos, G. H. (2005). Systematic Review in Software Engineering, COPPE/UFRJ, RT-ES 679/05.

Burégio, V. A., Almeida, E. S. and Meira, S. R. L. (2010). Characterizing dynamic software product lines - a preliminary mapping study. In Proc. of the 4th Int. Workshop on Dynamic Software Product Lines, pages 53-60.

Chen, L., Ali Babar, M. and Ali, N. (2009). Variability management in software product lines: a systematic review. In Proc. of the 13th Int. Soft. Product Line Conf., p. 81-90

Clements, P. and Northrop, L. (2001). Software Product Lines: Practices and Patterns. Addison-Wesley, $3^{\text {rd }}$ edition.

Galster, M. (2010). Describing variability in service-oriented software product lines. In Proc. of the 4th European Conf. on Software Architecture, pages 344-350.

Gimenes, I. and Travassos, G. H. (2002). "O Enfoque de Linha de Produto para Desenvolvimento de Software", In: XXI Jornada de Atualização em Informática Livro Texto, Editado pela Sociedade Brasileira de Computação, Porto Alegre.

Gimenes, I., Fantinato, M. and De Toledo, M. B. F. (2008). A product line for business process management. In Proc. of the 12th Int. Software Product Line Conf., pages 256-274. 
Gu, Q. and Lago, P. (2009). Exploring service-oriented system engineering challenges: a systematic literature review. Service Oriented Computing and Applications, v. 3, n. 3, pages 171-188.

Hallsteinsen, S., Hinchey, M., Park, S. and Schmid, K. (2008). Dynamic software product lines. Computer, v. 41, n. 4, pages 93-95.

Kang, D. and Baik, D. K. (2010). Bridging software product lines and service-oriented architectures for service identification using BPM and FM. In Proc. of the 9th IEEE/ACIS Int. Conf. on Computer and Information Science, pages 755-759.

Khurum, M. and Gorschek, T. (2009). A systematic review of domain analysis solutions for product lines. Journal of Systems and Software, v. 82, n. 12, pages 1982-2003.

Kitchenham, B. (2007). Guidelines for Performing Systematic Literature Reviews in Software Engineering, Keele University, EBSE Technical Report-2007-01.

Lee, J. and Kotonya, G. (2010). Combining Service-Orientation with Product Line Engineering. Software, IEEE, v. 27, n. 3, pages. 35-41.

Murugesupillai, E., Mohabbati, B. and Gašević, D. (2011). A preliminary mapping study of approaches bridging software product lines and service-oriented architectures. In Proc. of the 1st Workshop on Services, Clouds, and Alternative Design Strategies for Variant-Rich Software Systems, pages 1-8.

Northrop, L., Clements, P., Bachmann, F., et al. (2009). "A Framework for Software Product Line Practice, Version 5.0”, http://www.sei.cmu.edu/productlines/ framework.html, August 2011.

Papazoglou, M. P., Traverso, P., Dustdar, S. and Leymann, F. (2008). Service-oriented computing: a research roadmap. Int. Journal of Cooperative Information Systems, v. 17, n. 2, pages 223-255.

Petersen, K., Feldt, R., Mujtaba, S. and Mattsson, M. (2008). Systematic mapping studies in software engineering. In Proc. of the 12th Int. Conf. on Evaluation and Assessment in Software Engineering, pages 1-10.

Prieto-Diaz, R. and Freeman, P. (1987). Classifying Software for Reusability. IEEE Software, v. 4, n. 1, pages 6-16.

Sommerville, I. (2007). Engenharia de Software, Pearson Education, 8 edição.

Wieringa, R., Maiden, N., Mead, N. and Rolland, C. (2005). Requirements engineering paper classification and evaluation criteria: a proposal and a discussion. Requirements Engineering, v. 11, n. 1, pages, 102-107.

\section{Lista de Referências do MS (Corpus Reference)}

A lista dos estudos primários selecionados no MS está disponível no seguinte repositório: http://lpsoa.users.sourceforge.net 